\title{
AN EFFICIENT ADAPTIVE CONTROLLER DESIGN FOR THREE PHASE INDUCTION MOTORS BASED ON RBF NEURAL NETWORK
}

\author{
Erdal KILIÇ ${ }^{*}$, Sami ŞIT ${ }^{2}$, Hasan Rıza ÖZÇALIK ${ }^{2}$, Ahmet GANI ${ }^{2}$ \\ 1* Afşin Vocational School, Kahramanmaras Sutcu Imam University, Kahramanmaraş, Turkey \\ ${ }^{2}$ Department of Electrical Electronics Engineering, Faculty of Engineering, Kahramanmaras Sutcu Imam University, \\ Kahramanmaraş, Turkey \\ *Corresponding author; ekilic@ksu.edu.tr
}

Received: 11 November 2016; Accepted: 2 January 2017

\begin{abstract}
There are difficulties in control of induction motors which are widely used in industrial applications due to their nonlinear complicated structures. Nowadays the use of advanced control methods along with technological progress has made it possible to achieve high performance in the control of these motors. Thanks to its nonlinear and adaptive structure, artificial neural network based control algorithms will be a suitable and efficient method for control of induction motors. In this study, the speed control of three-phase squirrel cage induction motor was implemented using the dsPIC30F6010A microcontroller. In order to improve the performance of the drive system, a speed control algorithm has been developed using a radial basis function artificial neural network based model reference adaptive control method. The success of the proposed control algorithm has been experimentally tested by operating the induction motor under different speed and load conditions.
\end{abstract}

Key words: induction motor, vector control, artificial neural networks, model reference adaptive control, dsPIC microcontroller.

\section{GIRIŞ}

Asenkron motorların günümüz endüstrisinde yaygın kullanımı bu motorların sağlamlığı, yüksek verimliliği ve işletme güvenilirliğinden kaynaklanmaktadır. Bu motorlar aynı güçteki doğru akım motorları ile karşılaştırıldığında daha az bakım gerektirmekte, hacim ve ağırlık olarak daha küçük olmakla birlikte daha makul maliyetleri bulunmaktadır. Ancak karmaşık ve doğrusal olmayan yapıları nedeniyle asenkron motorları geniş bir yelpazede kontrol etmek oldukça zor olmaktadır. Bu nedenle kontrol hedeflerine ulaşmak güçlü ve sağlam kontrol algoritmaları yanında gelişmiş motor sürücü sistemleri de gerektirmektedir[1-5].

Asenkron motor sürücülerinde yüksek dinamik performansa sahip hız ve tork kontrol tekniklerini belirlemek amacıyla gerçekleştirilen birçok araştırma bulunmaktadır[6-11]. Bu çalışmalardan asenkron 
motor kontrol uygulamalarında performans gereksiniminin vektör kontrol tekniğiyle karşılanabildiği görülmektedir. Vektör kontrol yönteminde, uzay vektörleri kontrol algoritmasına göre işlenerek üç fazlı motorun gerilim ve akım anlık değerleri ayarlanır[12].

Asenkron motorların hız kontrolünde, genellikle basit yapılarından dolay1 geleneksel PI tipi denetleyiciler tercih edilmektedir. Ancak sistemin doğrusal olmayan yapısı ve değişen motor dinamiklerinin etkisi nedeniyle bu denetleyicilerden iyi bir performans elde edilememektedir[13]. Bu nedenle asenkron motor uygulamalarında adaptif, dayanıklı ve doğrusal olmayan denetleyiciler kullanılmaya başlanmıştır.

Model referans adaptif kontrol (MRAK), parametreleri bilinmeyen veya kısmen bilinen karmaşı doğrusal olmayan sistemlerin kontrolünde yaygın kullanılan adaptif kontrol yöntemlerindendir. Bazı ayarlanabilir denetleyici parametreleri ve bunları ayarlamak için bir ayarlama mekanizması ile doğrudan uyumlu bir kontrol sistemidir. Bu yöntemde, sistem çıkışının kararlılığı bilinen bir referans model çıkışını takip etmesi amaçlanır. Referans model, sistemde yükselme zamanı, maksimum aşım, kararlı hal hatası ile ilgili istenen özelliklere sahip ideal bir modeldir. Denetleyicinin performansı, uygun referans model seçimi ve ayar mekanizmasının yeteneğine bağlıdır[14-16].

Yapay sinir ağları (YSA) öğrenme ve adapte yeteneği sayesinde doğrusal olmayan dinamik sistemlerin modelleme ve kontrolünde yaygın olarak kullanılmaktadır[17-18]. Son yıllardaki çalışmalardan radyal taban fonksiyonlu yapay sinir ağları (RTYSA) asenkron motor sürücü sistemlerinde dayanıklılı̆g ve performansı artırdığı görülmektedir[19-21]. Bu çalışmada RTYSA sistemden gelen doğrusal olmayan kısımları kompanze etmek amacıyla denetleyici yapısında kullanılmıştır.

$\mathrm{Bu}$ çalışmada, asenkron motor sürücülerinde güvenilirliği ve verimliliği artırmak amacıyla RTYSA tabanlı MRAK bir denetleyici geliştirilmiştir. Bu denetim algoritması üç fazlı sincap kafesli $0.25 \mathrm{~kW}$ gücünde bir asenkron motorun hız kontrolünde deneysel olarak farklı hız ve yük durumları için test edilmiştir. Motorun sürme yönteminde yüksek performanslı asenkron motor sürücülerinde kullanılan dolaylı alan yönlendirmeli kontrol tekniği tercih edilmiştir. Üç fazlı eviricinin anahtarlamasında uzay vektör darbe genişlik modülasyon tekniği kullanılmıştır. Bu teknik yüksek hesaplama gücü gerektirdiğinden gelişmiş bir sayısal işaret işleyici olan dsPIC30F6010A mikrodenetleyicisi kullanılmıştır.

\section{YÖNTEM}

\subsection{RTYSA Yapısı}

RTYSA danışmanlı öğrenme algoritması kullanan çok katmanlı ileri beslemeli ağ mimarisine sahiptir. $\mathrm{Bu}$ ağ yapısı giriş, gizli ve çıkış olmak üzere üç farklı katmandan oluşmaktadır. RTYSA'da gizli katman aktivasyon fonksiyonu olarak genellikle doğrusal olmayan yapıya sahip Gauss Fonksiyonu kullanilır[22-23].

$$
\phi_{j}(x)=\exp \left[\frac{-\left\|x-c_{j}\right\|^{2}}{\sigma_{j}^{2}}\right]
$$

Burada $x$ giriş vektörünü, $c_{i}$ merkez vektörünü, $\| \mathrm{x}$-ci $\|$ standart Öklid uzaklığını, $\sigma_{j}$ radyal taban fonksiyon genişliğini göstermektedir. $c_{i}$ ve $\sigma_{j}^{\prime}$ ler giriş değerlerinin aralığına göre belirlenir. RTYSA'nın eğitimi adaptif parametrelerin $\left(c_{i}, \sigma_{j}\right.$ ve çıkış ağırlıkları $w_{k j}$ ) optimize edilmesi sürecidir[24-25].

Bu çalışmada Şekil-1'de gösterildiği gibi RTYSA topolojisi 2:5:1 (giriş katmanı düğüm sayısı 2, gizli katman düğüm sayısı 5 ve çıkış katmanı dügüm sayısı 1) ağ mimarisi yapısında seçilmiştir. 


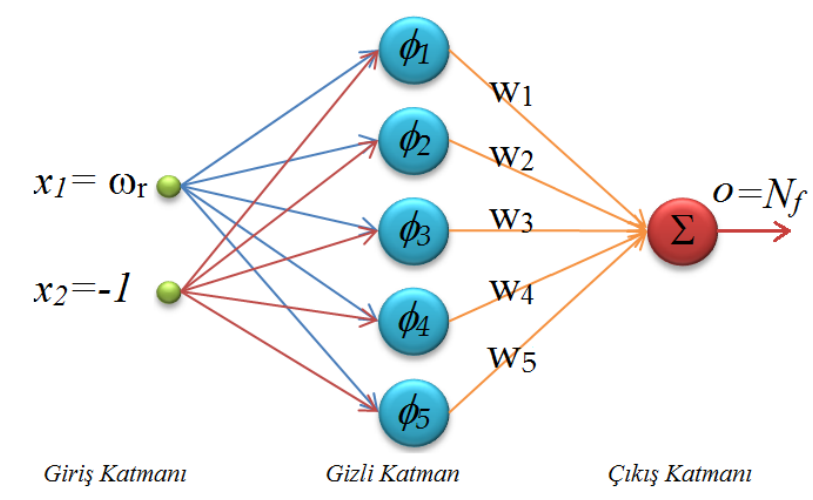

\section{Şekil 1. RTYSA Yapısı}

Ağ çıkışı aşağıdaki gibi ifade edilir:

$$
o_{k}=\sum_{j=1}^{J} w_{k j} \phi_{j}(x)
$$

Ayarlanabilen RTYSA parametreleri hatanın geri yayılım algoritması kullanılarak online ayarlanır.

$$
\begin{aligned}
& w_{k j}(t+1)=w_{k j}(t)+\eta e(t) \phi_{j} \\
& c_{j}(t+1)=c_{j}(t)+\eta e(t) \phi_{j} w_{j} \frac{\left(x-c_{j}\right)}{\sigma^{2}} \\
& \sigma_{j}(t+1)=\sigma_{j}(t)+\eta e(t) \phi_{j} w_{j} \frac{\left\|x-c_{j}\right\|}{\sigma^{3}}
\end{aligned}
$$

Burada $\eta(0,1)$ aralığında seçilen öğrenme katsayısıdır. $e(t)$ izleme hatasını göstermek üzere RTYSA'nın eğitilmesi için minimize edilmesi amaçlanan performans fonksiyonu aşağıdaki gibi ifade edilebilir.

$$
E(t)=\frac{1}{2} e(\mathrm{t})^{2}
$$

\subsection{Denetleyici Yapısı}

MRAK algoritması adaptif bir denetleyici tasarlanması için kullanılmıştır. Bu yöntem sistem çıkışının referans model çıkışını takip edebilmesi için kontrol parametrelerini ayarlama prensibine göre çalışmaktadır. Şekil 2'de gösterildiği gibi önerilen kontrol şeması referans model ve RTYSA olmak üzere iki ana bölümden oluşmaktadır[26]. Sistem ve referans model ile ilgili diferansiyel eşitlik aşağıdaki gibi ifade edilebilir:

$$
\begin{aligned}
& \dot{\omega(t)}+f[\omega(t)]=I_{s q}(t) \\
& \dot{\omega_{m}}(t)+k_{1} \omega_{m}(t)=k_{2} \omega_{r e f}(t)
\end{aligned}
$$

Burada $\omega(t)$ ve $\omega_{m}(t)$ sırayla sistem ve referans model çıkışları; $I_{s q}(t)$ kontrol sinyali; $f($.$) bilinmeyen$ statik doğrusal olmayan bir fonksiyon; $k_{1}$ ve $k_{2}$ pozitif katsayılardır. 


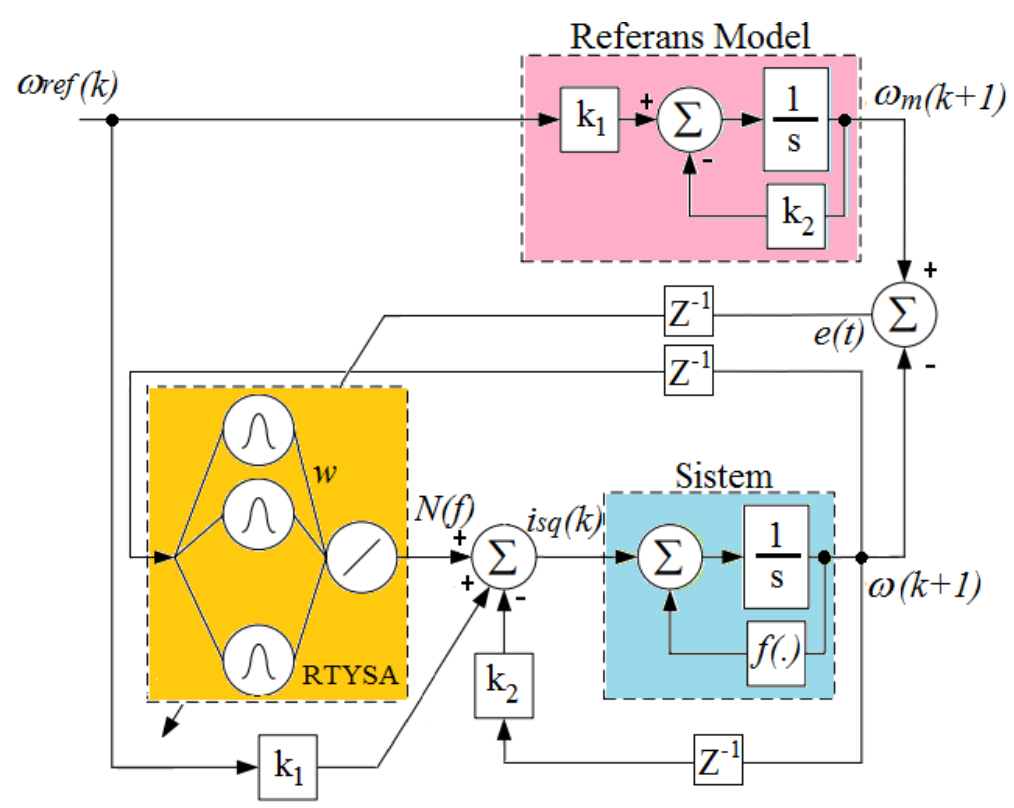

Şekil 2. RTYSA Tabanlı MRAK Yapısı

Model referans adaptif sistemin amacı kontrol edilen sistemin çıkışını model çıkışını izlemeye zorlayan bir denetleyici tasarlamaktır. Denetleyici tasarımı için, kontrol kuralı aşağıdaki gibi önerilebilir:

$$
I_{s q}(t)=-k_{2} \omega(t)+k_{1} \omega_{r e f}(t)+N_{f}[\omega(t), w(t)]
$$

Burada $N_{f}$ RTYSA çıkış vektörü; $w$ RTYSA ağırlık vektörüdür. A $\breve{g}$ çıkışı $N_{f}$ sistemin $f($.) ile ifade edilen doğrusal olmayan kısmını kompanze etmektedir. $N_{f}$ aşağıdaki gibi ifade edilir.

$$
N_{f}[\omega(t), w(t)]=\sum_{j=1}^{J} w_{j}(t) \exp \left[\frac{\left\|\omega(t)-c_{j}\right\|^{2}}{2 \sigma^{2}}\right]
$$

Eşitlik (7), (8) ve (9) birleştirilip yeniden yazılırsa aşağıdaki ifade elde edilir.

$$
\left[\dot{\omega(t)}-\dot{\omega_{m}}(t)\right]+k_{2}\left[\omega(t)-\omega_{m}(t)\right]=N_{f}[\omega(t), w(t)]-f[\omega(t)]
$$

Burada $N_{f}$ asimptotik olarak $f($.$) 'ye yaklaştığı zaman, izleme hatası e(t)$ sıfıra doğru eğilim gösterecektir.

$$
\begin{aligned}
& e(t)=\omega(t)-\omega_{m}(t) \\
& \dot{e}(t)+k_{2} e(t) \approx 0
\end{aligned}
$$

\subsection{Deney Düzeneği}

Deney düzeneği asenkron motorun hız kontrolünün gerçekleştirilmesi için tasarlanmıştır. Deney düzeneği dsPIC30F6010A mikrodenetleyici tabanlı motor kontrol geliştirme kartı, $1 \mathrm{~kW}$ doğrultucu ve evirici modülü, üç fazlı sincap kafesli $0.25 \mathrm{~kW}$ asenkron motor, yükleme amaciyla doğru akım şönt generatörü ve ölçüm cihazlarından oluşmaktadır. Asenkron motor sürücü sisteminin blok diyagramı Şekil 3'te, deney düzeneği ise Şekil 4'te gösterilmiştir. 


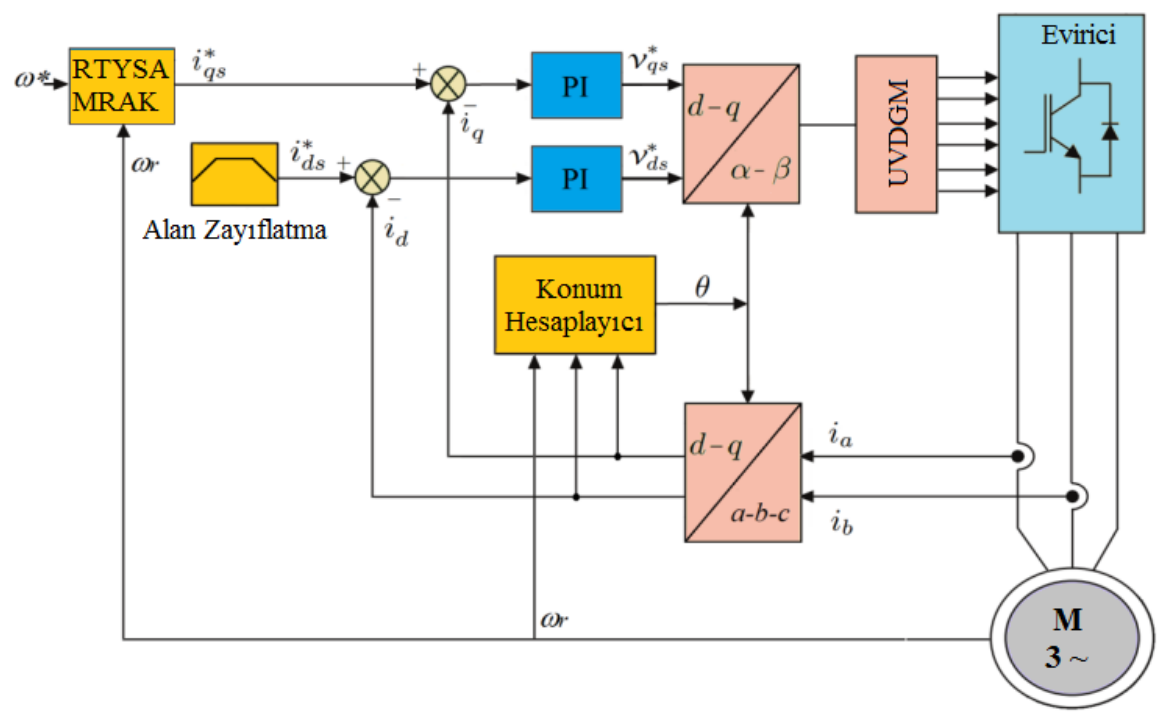

Şekil 3. Asenkron Motor Sürücü Sisteminin Blok Diyagramı

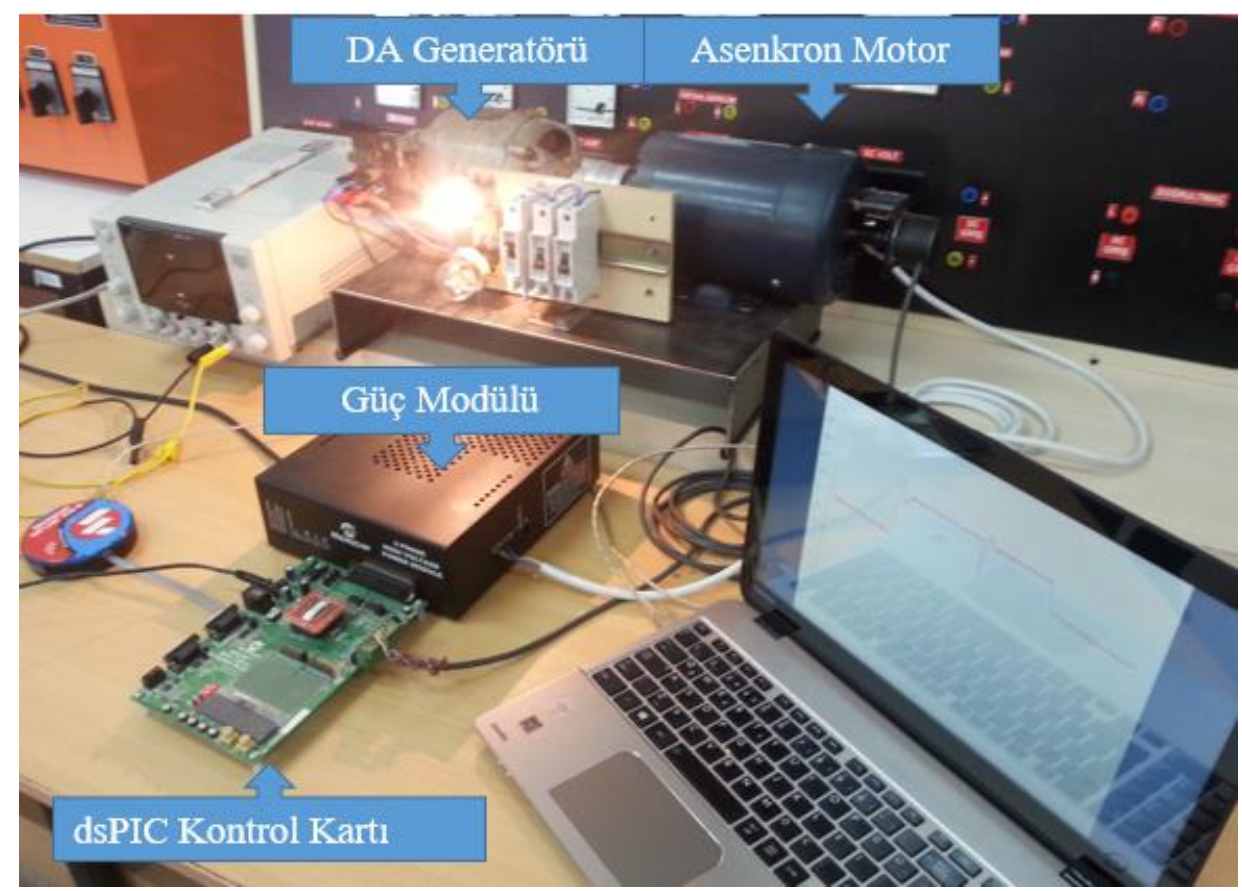

Şekil 4. Asenkron Motor Sürücü Sisteminin Deney Düzeneği

Kontrol sisteminde hız, akı ve torku kontrol eden üç kontrol döngüsü kullanılmaktadır. Önerilen RTYSA tabanlı MRAK hız kontrolörü olarak kullanılmıştır. Kontrol kartında akı ve tork kontrol döngüleri 100 $\mu s^{\prime}$ de, hız kontrol döngüsü ise 10 ms olarak yürütülmüştür. Eviricinin anahtarlama frekans1 $10 \mathrm{kHz}$, ölü zaman ise $2 \mu$ s seçilmiştir. Asenkron motor sürücünün sistem cevabı ani referans hız ve ani yük değişimleri için gösterilmiştir. 


\section{BULGULAR}

Asenkron motorun değişken hız referansları için elde edilen grafiğgi ve sistemin takip etmesi istenilen referans model çıkış işareti Şekil 5'te gösterilmiştir. Motora $1000 \mathrm{~d} / \mathrm{d}$ referans hız ile yüksüz olarak yol verilmiştir. Çalışmanın 5. saniyesinde referans hız değeri $1000 \mathrm{~d} / \mathrm{d}$ 'dan $1500 \mathrm{~d} / \mathrm{d}$ 'ya çıkarılmıştır. Çalışmanın 15. saniyesinde referans hız değeri 1500 d/d'dan 1200 d/d'ya düşürülmüştür. Referans tork karakteristiğini temsil eden hız denetleyicisinin ürettiği kontrol işareti Şekil 6'da gösterilmiştir.

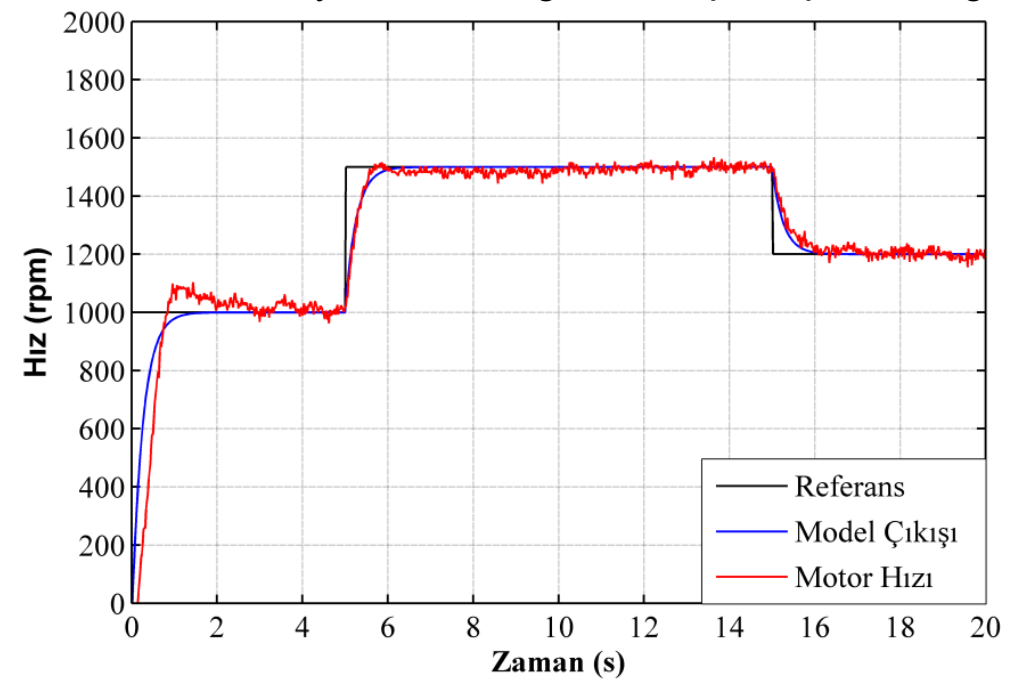

Şekil 5. Asenkron Motor Referans Hız Değişimlerindeki Hız Cevabı

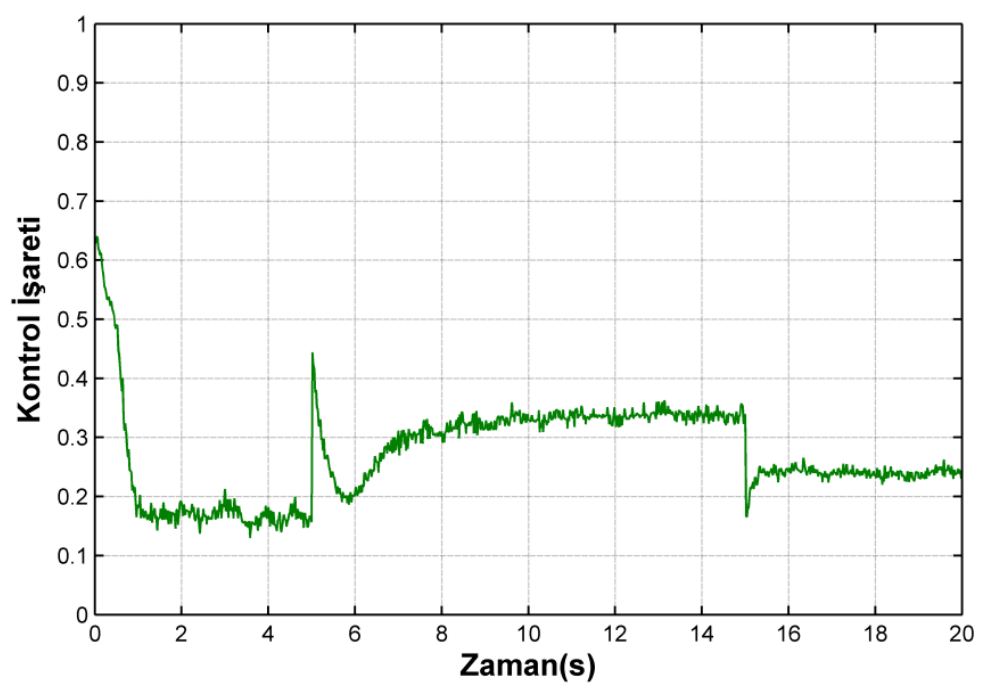

Şekil 6. Asenkron Motor Referans Hız Değişimlerindeki Kontrol Işareti 


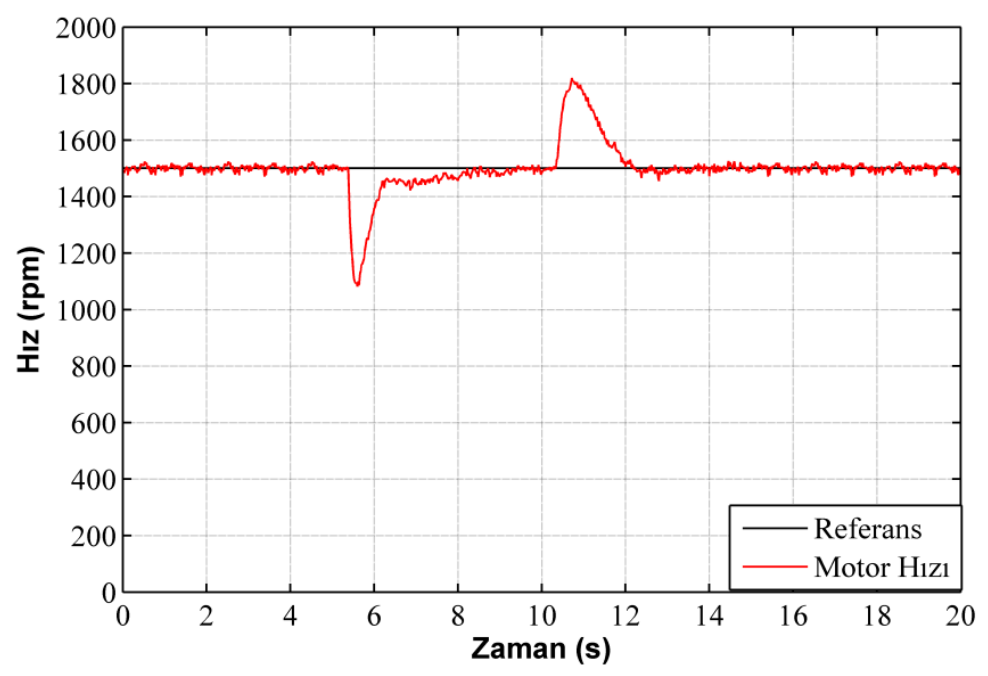

Şekil 7. Asenkron Motor Referans Yük Değişimlerindeki Hız Cevabı

Asenkron motor $1500 \mathrm{~d} / \mathrm{d}$ referans hızda kararlı durumda çalışırken doğru akım generatörüne bağlı lamba yükü devreye alınmıştır. Bu yüklenme durumu asenkron motorun hızında anlık düşüşe sebep olmuştur. Denetleyici motor hızını tekrar kararlı duruma getirmiştir. Yine motor kararlı durumda çalışırken lamba yükü devreden çıkarılmıştır. Bu durum motorun hızında anlık yükselme etkisi oluşturmuştur. Denetleyici etkiyi telafi ederek motorun istenilen hızda çalışmasına devam etmesini sağlamıştır. Yük değişimi altında elde edilen hız grafiği Şekil 7'de gösterilmiştir. Bu çalışma durumunda üretilen kontrol işareti Şekil 8'de gösterilmiştir.

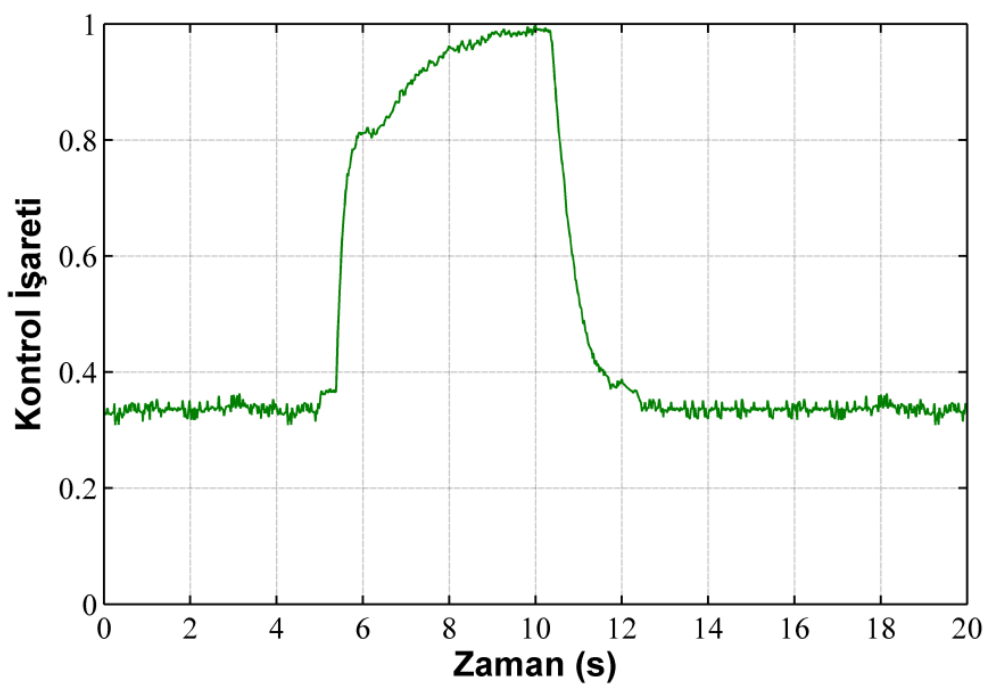

Şekil 8. Asenkron Motor Referans Yük Değişimlerindeki Kontrol Işareti

\section{SONUÇ}

Bu çalışmada, dsPIC30F6010A mikrodenetleyici tabanlı asenkron motor hız kontrol sistemi RTYSA tabanlı MRAK denetleyici kullanılarak gerçekleştirilmiştir. Dolaylı alan yönlendirmeli kontrol tekniğinde hız denetleyicisi olarak genellikle kullanılan PI denetleyici yerine RTYSA tabanlı MRAK denetleyici kullanılmışır. Önerilen denetim algoritmasının performansı farklı çalışma koşullarında deneysel verilerle sunulmuştur. 
Motora ait hız grafikleri incelendiğinde referans hızın değişim bölgelerinde sistemin istenilen değerler kısa sürede ulaştığı, aşmanın küçük olduğu ve sürekli hal hatasının oldukça küçük kaldığı görülmektedir. Yük değişiminin motor hızında oluşturduğu bozucu etki denetleyici tarafından kısa sürede ve yumuşak geçişle düzeltilmiştir. RTYSA tabanlı MRAK denetim yönteminin, yüksek dinamik performans gerektiren vektör kontrollü asenkron motor sürücülerinde kullanılması önerilmiştir.

\section{KAYNAKLAR}

1. Açıkgöz, H., \& Şekkeli, M. (2013). Bulanık Mantık Denetleyici ile Doğrudan Moment Denetim Yöntemi Uygulanan Asenkron Motorun Hız Denetim Performansının Incelenmesi. Akademik Platform Mühendislik ve Fen Bilimleri Dergisi, 1(2), 50-57.

2. Daya, J. F., \& Subbiah, V. (2012). A novel wavelet-fuzzy-based controller for robust speed control of induction motor drives. Australian Journal of Electrical and Electronics Engineering, 9(2), 185-195.

3. Mannan, M. A., Murata, T., Tamura, J., \& Tsuchiya, T. (2006). A fuzzy-logic-based self-tuning PI controller for high-performance vector controlled induction motor drive. Electric Power Components and Systems, 34(4), 471-481.

4. Özçalık, H. R., Yıldız, C., Danacı, M., \& Koca, Z. (2007). RBF based induction motor control with a good nonlinearity compensation. Paper presented at the International Work-Conference on Artificial Neural Networks.

5. Ustun, S. V. (2007). GA-based optimization of PI speed controller coefficients for ANNmodelled vector controlled induction motor. Applied Sci, 7, 4001-4006.

6. Kılıç, E., Özçalık, H. R., Yılmaz, Ş., \& Şit, S. (2015). A comparative analysis of FLC and ANFIS controller for vector controlled induction motor drive. Paper presented at the 2015 Intl Aegean Conference on Electrical Machines \& Power Electronics (ACEMP), 2015 Intl Conference on Optimization of Electrical \& Electronic Equipment (OPTIM) \& 2015 Intl Symposium on Advanced Electromechanical Motion Systems (ELECTROMOTION).

7. Kim, D. H. (2007). GA-PSO based vector control of indirect three phase induction motor. Applied Soft Computing, 7(2), 601-611.

8. Lin, F.-J., Wai, R.-J., Kuo, R.-H., \& Liu, D.-C. (1998). A comparative study of sliding mode and model reference adaptive speed observers for induction motor drive. Electric Power Systems Research, 44(3), 163-174.

9. Menghal, P., \& Laxmi, A. J. (2013). Adaptive Neuro Fuzzy based dynamic simulation of induction motor drives. Paper presented at the Fuzzy Systems (FUZZ), 2013 IEEE International Conference on.

10. Ramesh, T., Panda, A. K., \& Kumar, S. S. (2015). Type-2 fuzzy logic control based MRAS speed estimator for speed sensorless direct torque and flux control of an induction motor drive. ISA transactions, 57, 262-275.

11. Vas, P. (1990). Vector control of AC machines (Vol. 22): Oxford University Press, USA.

12. Trzynadlowski, A. M. (2000). Control of induction motors: Academic press.

13. Şit, S., Özçalık, H. R., Kılıç, E., \& Yılmaz, Ş. (2015). Üç Fazlı Asenkron Motor Sürücülerinde Hız Kontrol Yöntemlerinin Araştırılması.

14. Al-Aubidy, K., \& Ali, M. (2004). A Hierarchical Neuro-Fuzzy MRAC of a Robot in Flexible Manufacturing Environment. Int. Arab J. Inf. Technol., 1(2), 209-214.

15. Jain, P., \& Nigam, M. (2013). Design of a model reference adaptive controller using modified MIT rule for a second order system. Advance in Electronic and Electric Engineering, ISSN, 2231-1297.

16. Prakash, R., \& Anita, R. (2012). Modeling and simulation of fuzzy logic controller-based model reference adaptive controller. International Journal of Innovative Computing, Information and Control, 8(4), 2533-2550.

17. Efe, M. Ö., \& Kaynak, M. O. (1997). Doğrusal Olmayan Dinamik Sistemlerin Yapay Sinir Ağlarl Tarafindan Taninmast ve Denetimi. Paper presented at the TOK'97 Endüstriyel Otomasyon Teknolojileri Sempozyumu, Mecidiyeköy, Istanbul, Türkiye. 
18. Kı1ıç, E., Özbalc1, Ü., \& Özçalık, H. R. (2012). Lineer Olmayan Dinamik Sistemlerin Yapay Sinir Ağları ile Modellenmesinde MLP ve RBF Yapılarının Karşılaştırılması. Paper presented at the ELECO '2012 Elektrik - Elektronik ve Bilgisayar Mühendisliği Sempozyumu, Bursa.

19. Brandstetter, P., Kuchar, M., \& Skuta, O. (2014). Implementation of RBF Neural Network in Vector Control Structure of Induction Motor. International Review of Electrical Engineering (IREE), 9(4), 749-756.

20. Qian, M., Pei, L., \& Hui-xian, H. (2009). Induction Motor Vector Control Based on Immune RBF Neural Network Sliding Mode Variable Control. Paper presented at the 2009 International Conference on Signal Processing Systems.

21. Şit, S., Kılıç, E., Özçalık, H. R., Altun, M., \& Gani, A. (2016). Model Reference Adaptive Control based on RBFNN for Speed Control of Induction Motors. Paper presented at the International Conference on Natural Science and Engineering (ICNASE'16), Kilis.

22. Haykin, S. (1999). Neural Networks: A Comprehensive Foundation. Upper Saddle River, New Jersey, USA.: Prentice Hall.

23. Zhang, M.-G., Li, W.-H., \& Liu, M.-Q. (2005). Adaptive PID control strategy based on RBF neural network identification. Paper presented at the 2005 International Conference on Neural Networks and Brain.

24. Okkan, U., \& Dalkılıç, H. Y. (2012). Radyal tabanlı yapay sinir ağları ile Kemer Barajı aylık akımlarının modellenmesi. Teknik Dergi, 23(112).

25. Qin, L., Zhou, X., \& Cao, P. (2012). New control strategy for PMSM driven bucket wheel reclaimers using GA-RBF neural network and sliding mode control. Elektronika ir Elektrotechnika, 122(6), 113-116.

26. Patino, H. D., \& Liu, D. (2000). Neural network-based model reference adaptive control system. IEEE Transactions on Systems, Man, and Cybernetics, Part B (Cybernetics), 30(1), 198-204. 